\title{
Analyse de la forme des tiges pour la construction des tarifs de cubage. Application au cèdre du Maroc (Cedrus atlantica Manetti)
}

\author{
O. M'HIRIT et J.G. POSTAIRE * \\ Ecole Nationale Forestière d'Ingénieurs, \\ B.P. 511, Salé, Maroc \\ *Faculté des Sciences, Universilé Mohamed $V$. \\ B.P. 1014, Rabat, Maroc \\ (Détaché du Centre d'Automatique de l'Université de Lille 1 , \\ F 59655 Villeneuve-d'Ascq Cedex)
}

\begin{abstract}
Résumé
Les équations de volume qui servent à la construction des tarifs de cubage sont essentiellement des relations statistiques qui lient les dimensions des arbres à la forme de leur tige. La construction de tarifs de cubage pour le cèdre du Maroc a permis de mettre en évidence le rôle fondamental que peuvent jouer les techniques de classification automatique et de reconnaissance des formes dans ce domaine de la foresterie.

Une analyse multidimensionnelle de la forme des tiges de plus de 2000 cèdres abattus a d'abord fait apparaître trois différents types de profil chez cet arbre. L'ensemble des massifs forestiers du Rif a alors été partagé en deux groupes au sein de chacun desquels ces types de profil présentent une relative stabilité. La comparaison des performances de tarifs généraux, valables pour toute l'aire de répartition du cèdre dans le Rif, à celles de tarifs spécifiques, calculés séparément pour ces deux groupes, a montré l'intérêt de ce partage. La précision des tarifs spécifiques a été ensuite sensiblement améliorée grâce à un échantillonnage stratifié, chaque strate étant exclusivement composée d'arbres représentatifs de l'un des trois types de profils.

Afin de chiffrer de manière objective l'apport de cette nouvelle méthodologie, les performances des différents tarifs ont été testées sur des lots représentatifs de toutes les cédraies du Rif. Les gains de précision et de fiabilité ainsi obtenus pour les tarifs à une et à deux entrées sont très encourageants. Ils montrent l'intérêt de procéder à une analyse multidimensionnelle de la forme des tiges avant de construire tout tarif de cubage, quelle que soit la méthode utiliséc.
\end{abstract}

\section{Introduction}

La construction des tarifs de cubage est certainement l'un des premiers problèmes auxquels les forestiers ont été confrontés (Mendiboure \& Pronier, 1971). Pour des raisons évidentes, il est en effet exclu d'envisager la mesure directe du volume de chaque tige d'un peuplement. Pour des forêts à pénétration aisée, la solution généralement 
adoptée consiste à prélever un échantillon, sur lequel il sagit de mettre en évidence les relations statistiques qui lient le volume des tiges à des grandcurs mesurées sur les arbres, telles que le diamètre à hauteur dhomme, la hauteur totale et parfois une mesure de la forme (Bruce et al., 1968). Lapplication de ces relations aux arbres de tout un peuplement doit permettre destimer le volume sur pied avec précision.

Les tarifs de cubage sont généralement obtenus par régression, à partir d'échantillons qui doivent refléter aussi fidelement que possible les caractéristiques dendrométriques des arbres atxquels ils sont destinés (BOuCHON, 1974). Une très grande attention doit done être porlée à la manière de conduire l'échantillonnage. En effet, il est vain de faire appel à des outils mathématiques sophistiqués et de mettre en œuvre de puissants moyens de calcul pour construire un tarif de cubage si les arbres de l'échantillon utilisé ne représentent pas de manière satisfaisante les propriétés morphométriques des arbres des forêts à cuber. Cest à cette question fondamentale du choix des arbres-échantillons que cet article est consacré car, avec Brenac (1958), nous pouvons affirmer que « la méthode statistique ne saurait fournir une précision qui serait absente des donnécs quion lui propose d"interpréter».

Un tarif de cubage doit, en fait. lenir compte des variations naturelles des propriétés morphométriques des tiges des peuplements auxquels il est destiné. Or lensemble des mécanismes et des facteurs responsables de la forme de la tige d'un arbre est extrêmement complexe. On sait que le contexte bioclimatique, l'environnement de larbre, sa position sociale au scin du peuplement et ses possibilités de nutrition influencent sa forme (Boucrion, 1974). Les variations de forme sont également liées aux contraintes mécaniques subies par larbre. telles que le poids des branches (HoHENADL, 1924), leffot du vent (Jonson, 1912), de la neige, etc. Même des variations dordre génétique ne sont pas exclues (Mradibovre. 1971 ).

Pour tenir compte de tous ces facteurs responsables de la grande variabilité naturelle de la forme des tiges, de nombreux auteüs ont décrit le profil des arbres par ajustement dune courbe (Fris: \& MATERN. 1965: NASIUND, 1980) ou d'une équation analytique (LArson, 1963: PArm. 1981; Ko\%ak 't al., 1969). Mais les retombées pratiques de ces études sont souvent restées limitées, tout au moins au niveau de la construction des tarifs de cubage. En effet. lexploitation de cette approche se limite généralement à la recherche dune forme moyenne (Mendibourli, 1971) et ne permet pas, te cas échéant, de mettre en évidence p'usieurs types de forme dans les peuplements considérés.

Une approche plus prometteuse, en ce sens quielle ne masque pas la grande variabilité des profils au sein des peuplements, consiste à classer les arbres par type de forme, sur la base d'un paramètre calculé à partir de mesures dendrométriques (BeHre, 1927 ; NasLund, 1980). Le paramètre le plus utilisé est sans doute le «coefficient de forme»f qui s'exprime par la formule:

$$
f=\frac{v}{g \cdot h}
$$

où $\mathrm{v}$ est le volume exact de la tige, g la surface terrière de l'arbre à $1,30 \mathrm{~m}$ et $\mathbf{h}$ sa hauteur totale (PARDE, 1961). D’autres auteurs préfèrent caractériser la forme des tiges par une relation entre lcs diamètres à deux niveaux différents (DecourT, 1965 ; NASLUND, 1980). 
Cependant, il paraît difficile d'envisager une étude de la variabilité des formes en se limitant à les enfermer dans un seul paramètre, aussi représentatif soit-il. En effet, une forme n'est pas un rapport unique : c'est un ensemble de rapports dont scules les techniques danalyse multidimensionnelle permettent de cerner les variations (BENZECRI, 1978). Le champ d'application de ces techniques, qui ne cesse de s'étendre, n’a pas encore atteint ce domaine de la foresterie, ce qui explique labsence de résultats déterminants dans les études sur les variations morphométriques des arbres (BOUCHON, 1974).

Nous pensons pourtant quiil est fondamental, avant de construire un tarif de cubage, de procéder à une analyse aussi fine et précise que possible de la forme des tiges des arbres considérés. Pour ce faire, nous proposons une technique de mesure qui permet de caractériser le profil d’un arbre par un ensemble de quatre paramètres (voir $\S 2,2$ ).

Cette caractérisation simple de la forme des tiges permet de faire appel à des techniques de classification automatique de type statistique pour analyser les variations morphométriques des arbres des peuplements étudiés (voir § 2.3).

Ces techniques danalyse multidimensionnelle, nouvellement mises au point par l'un des auteurs, sont appliquées à l'étude du cèdre du Maroc. Un polymorphisme très stable et très marqué est ainsi découvert chez les cèdres provenant de différents massifs forestiers du Rif et du Moyen Atlas (voir $\S 3$ ).

Les différences de forme mises en évidence sont suffisamment importantes pour quiil soit nécessaire de les faire intervenir lors de l'établissement des tarifs de cubage. Un plan d'échantillonnage tenant compte des différents types de profils rencontrés est proposé. Son intérêt pratique est illustré par la construction de différents tarifs de cubage pour les cédraies du Rif (voir \$ 4).

L'amélioration sensible de la précision et de la fiabilité des tarifs ainsi obtenus constitue un critère objectif pour apprécier l'apport des techniques de classification automatique et de reconnaissance des formes dans le domaine de la foresterie.

\section{Méthode d'étude des profils des tiges du cèdre du Maroc}

\subsection{Récolte des données}

Les arbres étudiés sont des cèdres provenant de huit grands massifs forestiers répartis entre le Rif et le Moyen Atlas (cf. fig. 1).

Les tiges de plus de 2000 cèdres provenant de différentes coupes de régénération et d'éclaircie ont été cubées par la méthode de Huber au cours de campagnes d'exploitation dans ces massifs forestiers. Ces tiges ont également été soumises à des mesures précises de diamètre et de hauteur.

Le arbres retenus, après rejet des individus aberrants, sont répartis entre les huit massifs forestiers comme indiqué sur le tableau 1. Les massifs de Tizirène et de Bab Chiker appartiennent au Rif Occidental, caractérisé par un climat humide et frais et un substrat de schistes et de grès. Les forêts de Ghommara, Tidighine, Beni Khennous et Jbcl Lerz font partie du Rif Central, humide et froid avec un substrat de grès 
et de quartzite de l'Albo-aptien. Quant aux massifs du Moyen Atlas, celui d'Ajdir est situé sur une assise de roches calcaires et de dolomics du Lias et du Jurassique, alors que celui de Kerrouchen est caractérisé par un substrat non carbonaté, en grande partie constitué de grès de rAutunien. Ces deux massifs bénéficient d'un climat humide et froid.

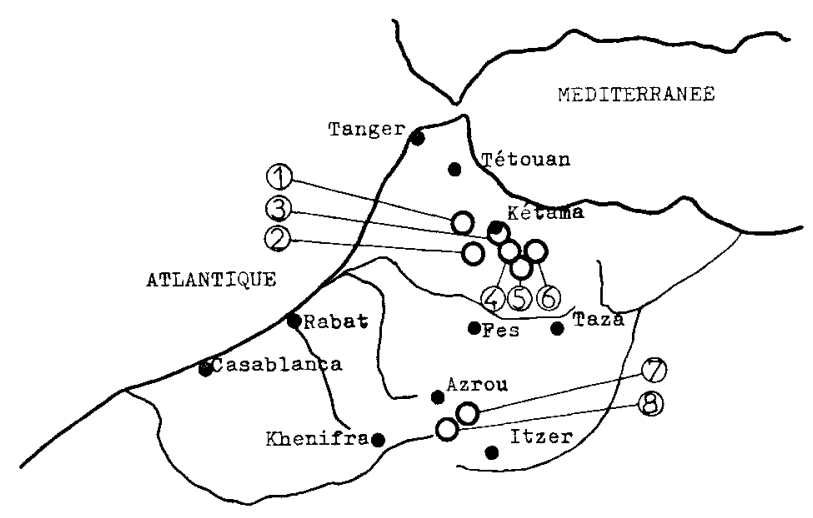

FIC; 1

Répartition géographique des huit massifs forrestiers étudicis.

Geographic position of the eight forest areas studied in Morocen.

Echelle : $1 / 5000000$.

(i) — $\longrightarrow$ : localisation du massif $\mathbf{n}^{\prime \prime} \mathbf{j}$.

\section{TABLEAU 1}

Caractéristiques des massifs forestiers du Rif et du Moyen Atlas.

Specifications of the forest areas of the Rif mountains and the Middle Atlas in Morocco.

\begin{tabular}{|c|c|c|c|c|}
\hline$-=\quad-$ & 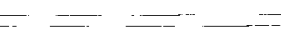 & $-\cdots$ & $=\quad=$ & 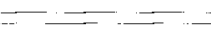 \\
\hline $\begin{array}{l}\mathrm{N}^{0} \text { d'ordre } \\
\text { affecté aul } \\
\text { massif forestier }\end{array}$ & $\begin{array}{l}\text { Nom } \\
\text { du massif foresticr }\end{array}$ & $\begin{array}{l}\text { Superficic } \\
\text { (ha) }\end{array}$ & $\begin{array}{l}\text { Altitude } \\
\text { moyenne } \\
(\mathrm{m})\end{array}$ & $\begin{array}{c}\text { Taille } \\
\text { de léchantillon } \\
\text { disponible }\end{array}$ \\
\hline 1 & Tizirèroc & 1327 & 1700 & 194 \\
\hline 2 & Bab Chiker & 740 & 1700 & 191 \\
\hline 3 & Ghommara & 2320 & 1650 & 154 \\
\hline 4 & Tidighine & 1850 & 2000 & 432 \\
\hline 5 & Beni Khennous & 1200 & 2000 & 356 \\
\hline 6 & Jbcl Lerz & 2400 & 1850 & 171 \\
\hline 7 & Ajdir & 38088 & 1900 & 377 \\
\hline 8 & Kerrouchen & 10840 & 1800 & 207 \\
\hline
\end{tabular}




\subsection{Caractérisation des profils}

Pour caractériser la forme des tiges, nous avons repris une idée déjà ancienne qui consistait à classer les arbres par «types denưrométriques» (PARdé, 1961). La méthode revenait à assimiler chaque tige à un solide de révolution constitué de portions de cylindres, de parabolö̈des, de cônes et de nélö̈des. Cette approche, dont la mise en cuvre très lourde a limité l'application pratique, nous a suggéré d'assimiler les tiges à un assemblage de trois trones de cône de révolution (cf. fig. 2). Le premier représente l'mpattement de larbre, c'est-à-dire la partie située entre le sol et 1,30 m. Le second permet de décrire la tige entre $1,30 \mathrm{~m}$ et la mi-hauteur $\mathrm{h} / 2$. Le troisième, enfin, représente la moitié supérieure de la tige.

Ces trois trones de cône permettent de caractériser directement la forme des tiges à partir de mesures couramment utilisées en foresteric, à savoir :

- Le diamètre de la souche $\left(\mathrm{d}_{\mathrm{s}}\right)$;

- Le diamètre à hauteur d'hommes (d):

- Le diamètre à mi-hauteur $\left(\mathrm{d}_{\mathrm{h} / 2}\right)$;

- I a hautcur totale (h).

Afin déliminer le factcur taille et dans le but d'assurer la reconstitution de la forme schématique proposée à partir des paramètres qui la définissent, nous avons retenu, pour caractériser chaque tige, les trois paramètres suivants :

- Le coefficient de décroissance :

$$
\delta=\mathrm{d}_{1 / 2 / 2} / \mathrm{d}
$$

- I_c coefficient d'empattement :

- L’angle de défilement :

$$
\varepsilon=\mathrm{d} / \mathrm{d}_{\mathrm{s}}
$$

$$
0=\operatorname{Arctg} \frac{\mathrm{d}-\mathrm{d}_{\mathrm{h} / \mathrm{z}}}{\mathrm{h}-2,6} \quad \text { (en radians). }
$$

Le cocfficient de décroissance ${ }^{(*)} \delta$, proposé par SCHIfFEL (1905), renseigne sur la forme de la moitié inféricure de la tige, alors que le coefficient d'empattement indique la forme de la base de larbre. La tangente de langle 0 caractérise la décroissance métrique sur le diamètre (*) depuis la hauteur d'homme jusqu'au milieu de l'arbre.

Pour nuancer cette schématisation de la forme qui peut paraître un peu grossière, nous adjoindrons à ces trois paramètres le coefficient de forme $\mathrm{f}$ défini précédemment, c'est-à-dire :

$$
f=\frac{4 v}{\pi \cdot d^{2} \cdot h}
$$

Ce cocfficient donne une indication sur la forme globale de la tige de la base jusqu'au sommet (Loetsci et al., 1973). Sa détermination nécessite la connaissance du volume exact des tiges qui a été déterminé par la méthode de Huber à partir du cubage de billons successifs.

(*) Norme : NF B 53017. 
Notons finalement que les quatre paranètres qui caractérisent la forme de chaque tige peuvent être obtenus sans difficulté, puisqü̈ls ne nécessitent que des mesures couramment effectuées sur le terrain. Cette méthode de caractérisation de la forme des tiges va nous permettre de procéder maintenant à leur analyse fine par l'emploi de techniques de reconnaissance des formes.

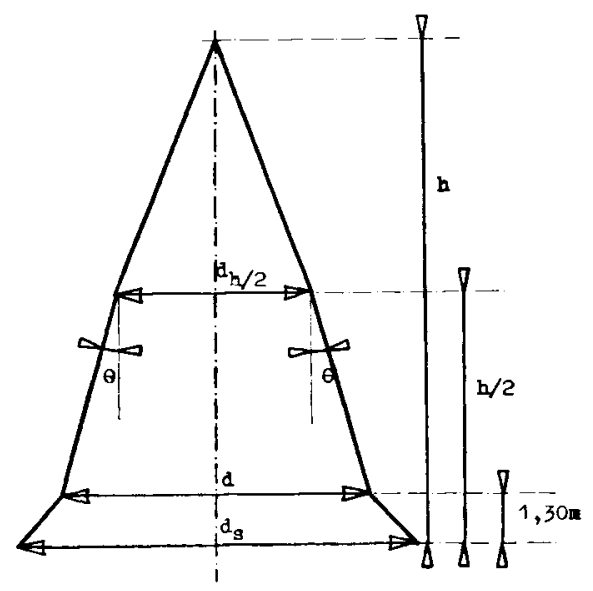

FIG. 2

Schématisation de la forme d'une tige par assemblage de trois troncs de cône de révolution. Shape description of a tree stem.

\subsection{Méthode d'analyse de la forme des profils}

Supposons que l'on dispose d'un échantillon constitué d’arbres tirés aléatoirement d'un peuplement et quà la forme de la tige de chaque arbre on associe l'observation quadridimensionnclle constituée par les quatre paramètres définis précédemment.

La forme de chaque tige, caraciérisće par son coefficient de décroissance $\delta$, son coefficient de forme $\mathrm{f}$, son angle de défilement $\theta$ et son coefficient dempattement $\varepsilon$ peut alors être représentéc par un point dans un espace à quatre dimensions.

Cette caractérisation de lá forme des tiges permet daborder lanalyse morphométrique du cèdre par des méthodes d'analyse multidimensionnelle. En effet, lapplication des techniques de classification autonatique à ces observations quadridimensionnelles doit permettre d'établir, si elles existent, la présence de différentes classes au sein des échantillons extraits des forêts considérées, chaque classe correspondant à un type de forme particulier.

On peut supposer que les observations collectées proviennent dune ou de plusieurs sources aléatoires de telle sorte que le problème de leur classification se trouve posé en termes statistiques. La distribution des observations peut être assimilée, dans ces conditions, à un mélange pondéré des fonctions de densité de probabilité rclatives aux différentes classes en présence. Le cocfficient de pondération de chaque fonction de densité n’est alors autre que la probabilité dapparition des individus de la classe correspondante. 
La connaissance du nombre de classes et, pour chacune delles, de sa fonction de densité de probabilité et de sa probabilité a priori, permet de classer des observations provenant d'un tel mélange de classes avec le taux d'erreur optimal, en dessous duquel le chevauchement des elasses ne permet théoriquement pas de descendre (Duda \& HAFi, 1973).

Mais dans le contexte de lanalyse morphométrique des tiges d'une essence forestière, les données nécessaires pour effectuer cette classification à taux d’erreur optimal ne sont pas dircetement disponibles. Il est toutefois possible de compenser ce manque de connaissance sur le mélange par l'information apportée par les observations à classer elles-mêmes. En effet, en supposant que les fonctions de densité des différentes classes sont des fonctions normales, le problème se trouve posé en termes d’analyse des mélanges gaussiens. Ce problème a ćté récemment résolu en analysant la convexité de la fonction de densité sous-jacente à la distribution des observations. Il a été montré que cette nouvclle approche permet de détecter les classes en présence et de déterminer des valeurs approchées de leurs vecteurs moyennes, matrices de covariance et probabilités a priori.

On dispose ainsi de valeurs approchées de tous les paramètres du mélange analysé, ce qui permet d'envisager une classification optimale par minimisation du taux d'erreur. Les fondements thćoriques de cette approche et ses modalités dimplantation sur calculateur numérique sont exposés en détail par ailleurs (Postalre \& Vasseur, 1981; Postaire, 1981. 1982 ; Postaire \& Vasseur, 1982).

Nous allons maintenant montrer comment ces techniques de classification automatique, utilisécs conjointement avec la méthode de caractérisation des profils décrite précédemment, permettent daborder lanalyse morphométrique du cèdre du Maroc comme un problème de reconnaissance des formes.

\section{Mise en évidence de différents types de profil}

\subsection{Polymorphisme des cèdres de chacule massif forestier}

Les huit massifs forestiers objets de cette étude ćtant bien individualisés sur le plan écologique, la forme des cèdres est d’abord analysée par massif.

On dispose, pour chaque massif, d'un échantillon dobscrvations quadridimensionnelles, chaque observation représentant le profil de la tige d'un cèdre du massif considéré. Nous supposerons implicitement que les observations suivent des lois normales. Comme on dispose de très peu dinformation a priori sur les phénomènes étudiés, la validité du choix de ce modèle ne sera justifiée qu'a posteriori, par le succès de l'utilisation qui en est faite.

Le premier résultat, fondamental, est la mise en évidence d'un polymorphisme marqué chez los cèdres étudiés. En effet, trois classes distinctes ont été détectées dans chacun des huit échantillons, les arbres assignés à chacune d'elles présentant le même type de profil. Dans chaque massif forestier, on met ainsi en évidence trois types de profils définis par les profils moyens des arbres de chacune des classes (cf. fig. 3).

Mais, présenté sous cette forme brute, ce résultat, qui fait intervenir 24 types de profil, reste d’un intérêt pratique limité. Il est cependant possible de synthétiser les résultats obtenus en analysant la stabilité des types de profil mis en évidence dans les différents massifs forestiers. 

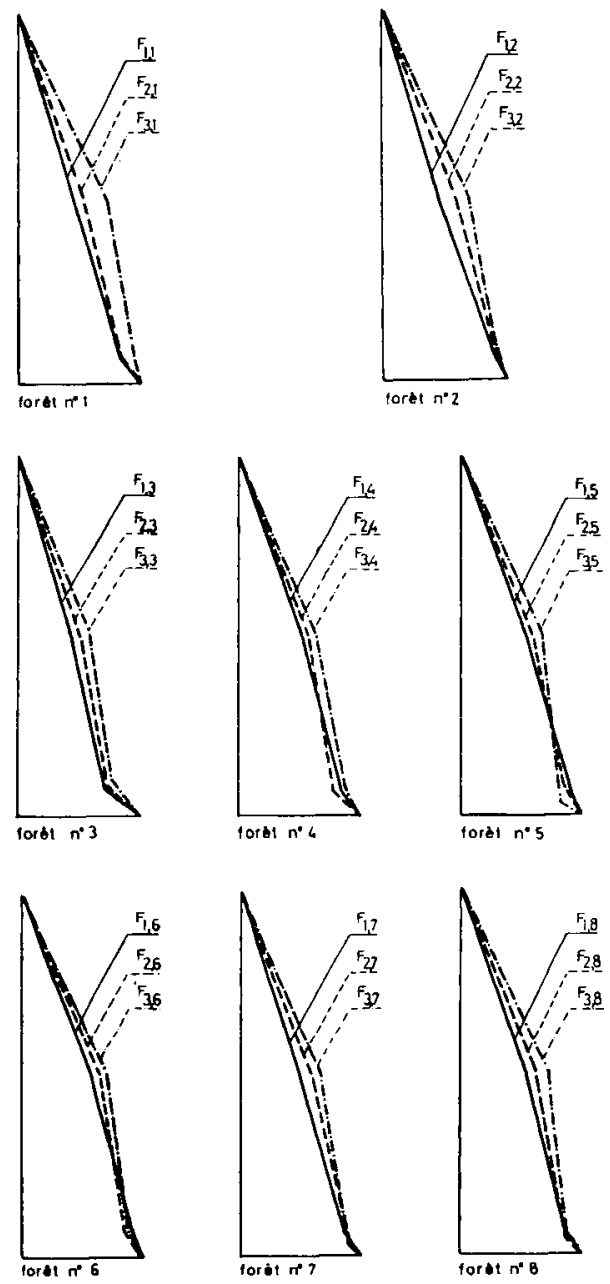

FiG. 3

Les trois types de profil mis en évidence dans chacun des huit massifs forestiers étudiés. The three stem patterns distinguished in each forest area.

\subsection{Stabilité des profils dans l'ensemble des massifs forestiers}

Les types de profil définis par lanalyse multidimensionnelle précédente sont notés $F_{i, j}$, i étant l'indice du type de profil au sein du massif forestier d'indice $j(i=1,2,3$; $\mathrm{j}=1,2, \ldots, 8$ ).

La stabilité du ième type de profil dans l'ensemble des massifs considérés peut être analysée en étudiant la répartition spatiale des points $F_{i . j}, j=1,2, \ldots, 8$ dans un espace quadridimensionnel. Nous ferons appel, pour cette analyse, à l'algorithme de recherche de groupements du Maximin (BATCHELOR \& Wilkins, 1969). Cet algorithme 
consiste dabord à rechercher les deux points les plus éloignés au sens de la distance euclidienne. Ceux-ci constituent les centres de deux premiers groupements. On affecte cnsuite chacun des points $F_{i . j}$ restants au groupement dont le centre est le plus proche, à condition toutefois que la distance de ce centre ne dépasse pas un certain seuil. Le cas échéant, si un point $F_{i, j}$ est trop éloigné des deux centres initiaux, il constitue le centre d'un nouveau groupcment et la procédure est réitérée.

La figure 4 indique le résultat de cette recherche de groupements sous la forme de graphes dont les nouds représentent les points $F_{i, j}$. Les longueurs des branches reliant les centres des groupements ou les points restants au centre le plus proche sont proportionnelles aux distances entre ces points dans l'espace à 4 dimensions.

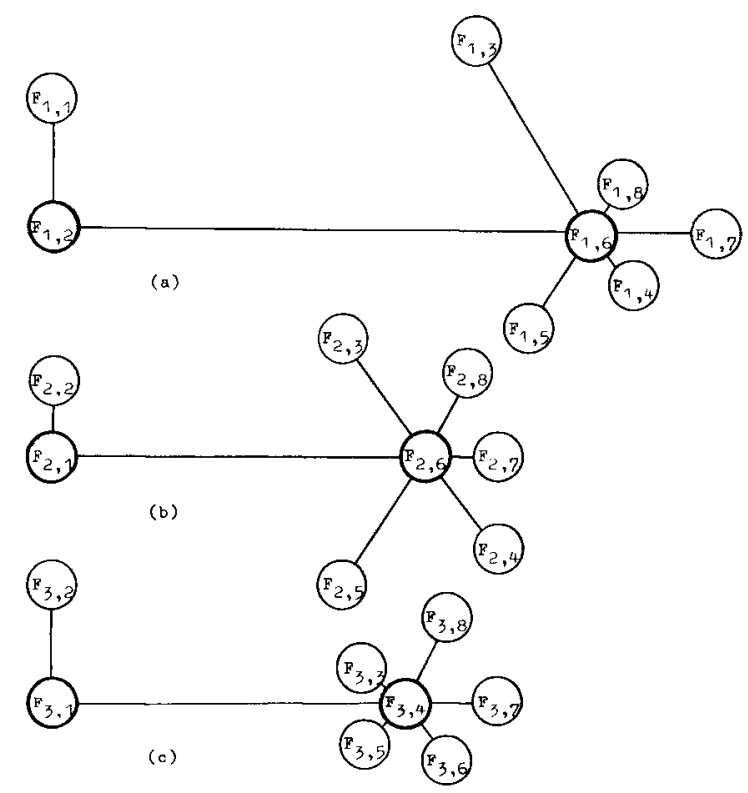

FIG. 4

Stabilité des trois types de profil mis en évidence dans les huit massifs forestiers étudiés.

Stability of the three stem patterns distinguished in each forest area.

Sur la base des groupements des points $F_{1, j}, j=1,2, \ldots, 8$, représentant le premier type de profil dans les huit massifs considérés, on constate qüil apparait deux ensembles de massifs. Le premier est constitué par les massifs $\mathrm{n}^{\prime \prime} 1$ et $\mathrm{n}^{\circ} 2$ pour lesquels les profils $F_{1.1}$ et $F_{1.2}$ sont très voisins. Le second ensemble regroupe tous les massifs restants pour lesquels les profils $F_{1 . j}, j=3,4, \ldots, 8$ sont très proches les uns des autres (cf. fig. 4 [a]).

Une analyse semblable, portant sur les points $F_{., j}, j=1,2, \ldots, 8$, représentant le second type de profil, conduit aux mêmes regroupements des massifs foresticrs (cf. fig. 4 [b]). 
Ces regroupements sont finalement confirmés par lanalyse de la répartition des points représentant le troisième type de profil dans les huit massifs étudiés (cf. fig. 4 [c]).

Cette analyse multidimensionnel!c met en relief une certaine stabilité des trois types de profil mis en évidence dans chacun des deux ensembles de massifs forestiers définis ci-dessous :

Ensemble $A$ constitué des massifs de :

- Tizirène $(n " 1)$;

- Bab Chiker (n"2);

Ensemble $B$ constitué des massifs de :

- Ghommara (1" 3) :

- Tidighine (n"4):

- Beni Khennous (n" 5);

- Jbel Lerz (n" 5);

- Ajdir (n"7) ;

- Kerrouchen (n" 8).
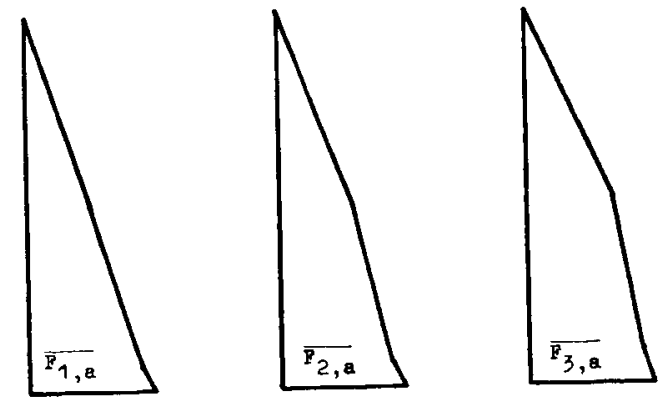

E N S E M B L E A
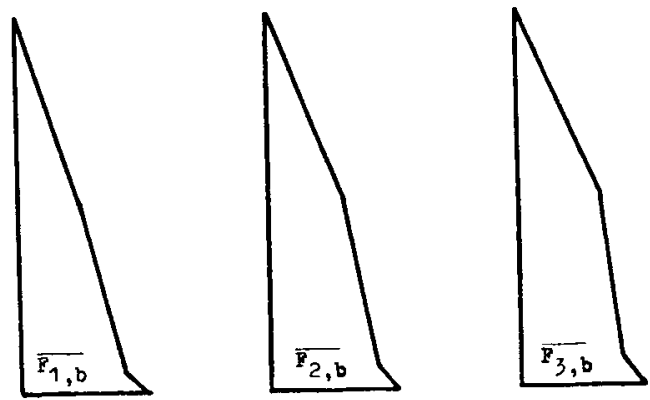

E N S E M B I E R

FIG. 5

Les trois protils-types mis en évidence dans l'ensemble $A$ et dans l'ensemble $B$.

The three average patterns distinguished in each group of forests. 
On peut ainsi définir des profils-types pour l'ensemble $A$ en calculant les barycentres $F_{i, a}$ des trois couples de points $\left\{F_{i, 1} ; F_{i, 2}\right\}, i=1,2,3$.

De la même manière, les barycentres $\overline{F_{i . b}}$ des trois groupements de points $\left\{F_{i .3}\right.$; $F_{i .4} ; F_{i .5} ; F_{i . f i} ; F_{i .7}: F_{i .8 i} i \mathrm{i}=1,2,3$, définissent trois profils-types pour l'ensemble $B$.

La figure 5 représente les trois prcfils-types ainsi mis en évidence dans chacun des ensembles $\mathrm{A}$ ct $\mathrm{B}$ de massifs forestiers.

Cette analyse de la forme des cèdres du Maroc montre que ceux-ci présentent un poiymorphisme marqué, qui ne peut être ignoré lors de la construction des tarifs de cubage. C'est dans ce sens que nous abordons maintenant le problème de léchantillonnage des arbres en vue de lamélioration de la précision et de la fiabilité des tarifs de cubage pour les cédraies du Rif.

\section{Etablissement des tarifs de cubage}

Nous nous proposons d'établir des tarifs de cubage à une entréc, le diamètre à hauteur d'homme, ainsi que des tarifs à deux entrées, le diamètre à hauteur d'homme et la hauteur totale (Rondeux, 1973). Nous al!ons construire ces deux types de tarifs pour l'ensemble A d'une part et pour l'ensemble B d'autre part. De ce dernier ensemble seront exclus les massifs forestiers d'Ajdir ( $n$ " 7) et de Kerrouchen ( $\left(n^{\prime \prime} 8\right.$ ) qui appartiennent au Moyen Atlas. Nous comparerons les performances de ces tarifs spécifiques à celles des tarifs généraux, à une et à deux entrées, calculés pour toutes les cédraies du Rif regroupées dans un troisième ensemble forestier : lensemble $C$.

\subsection{Tarifs établis par échantillonnage simple}

Une première série de tarifs est établie sur la base de données obtenues par échantillonnage aléatoire simp!e parmi les arbres déjà cubés, mesurés et utilisés pour l'analyse morphométrique. Les tirages sont effectués à l'aide d’un programme de génération de nombres aléatoires. De plus, comme il est dusage, les tirages sont conduits de manière à ce que les distributions des diamètres à hauteur dhomme pour les arbres des échantillons soient identiques à celles de ces diamètres dans les ensembles forestiers correspondants (Bouchon, 1974).

Le nombre de cèdres à tirer pour constituer l'échantillon représentatif de chaque ensemble forestier est prédéterminé en fonction de la précision souhaitée pour les tarifs. En limitant lerreur à craindre à \pm 10 p. 100 au seuil de signification de 95 p. 100 , un calcul simple permet de déterminer ce nombre à partir d'une estimation de la dispersion des volumes dans l'ensemble forestier considéré (Cochran. 1963).

\subsubsection{Calcul des tarifs}

Notre propos n'étant pas la recherche de l'équation la plus appropriée, nous nous sommes arrêtés à la construction des tarifs à une entrée les plus utilisés en France (Abadie \& Ayral, 1956), en Angleterre (Hummel, 1955) et qui trouvent atuelques adeptes en Allemagne (DitTmar, 1958), à savoir :

$$
\hat{\mathrm{v}}=\mathrm{a}_{0}+\mathrm{a}_{1} \mathrm{~d}^{2}
$$




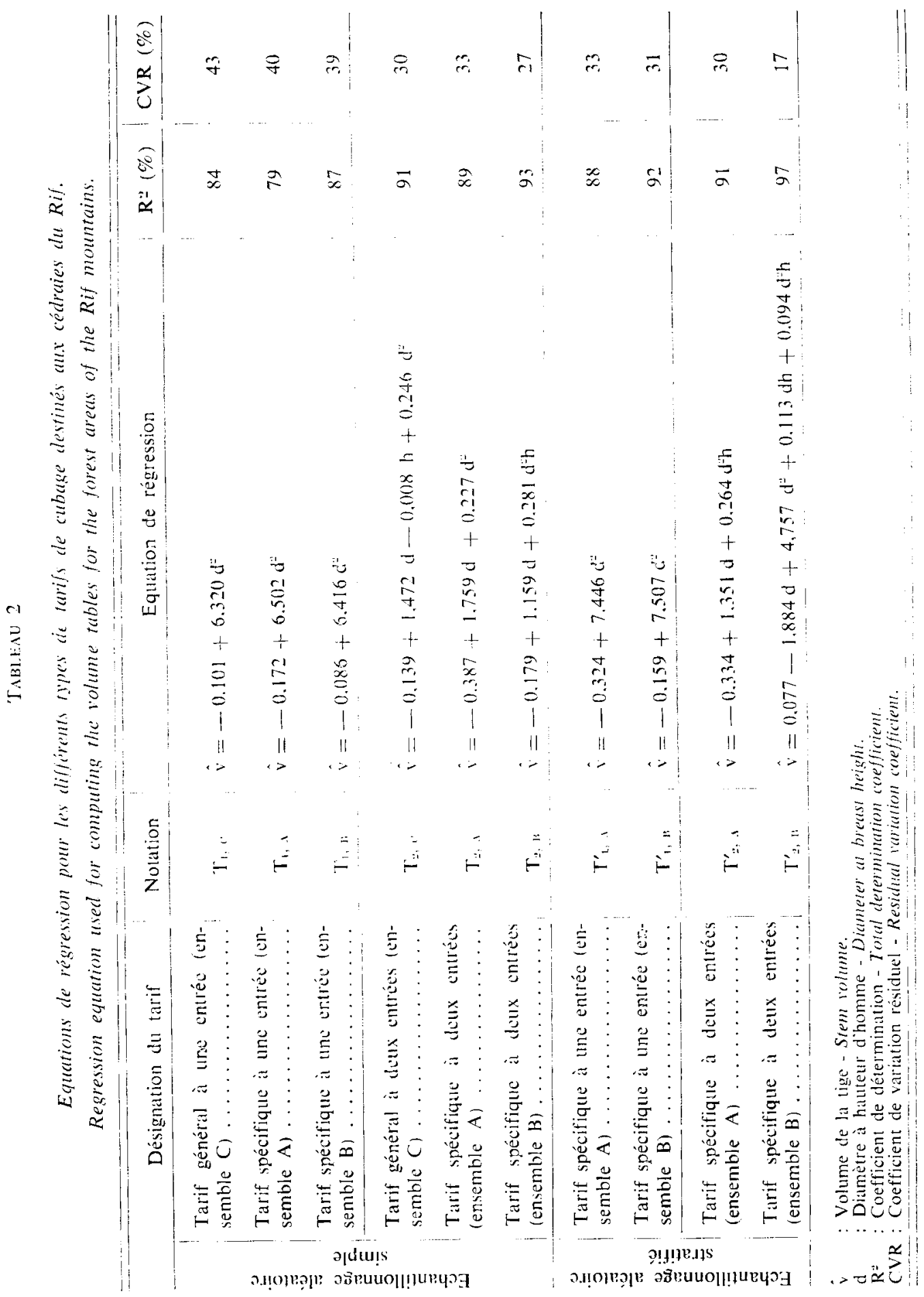


Cette équation, ajustée par régression aux données de chacun des échantillons tirés des ensembles forestiers $A, B$ et $C$ donne respectivement les tarifs $T_{1, A}, T_{1, B}$ et $T_{1, C}$ du tableau 2.

Les tarifs à deux entrées, la hauteur h et le diamètre $\mathrm{d}$, ont été obtenus sur la base des mêmes échantillons que ceux utilisés pour les tarifs à une entrée, en ajustant un

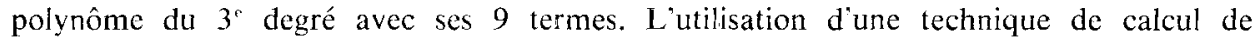
régression multiple pas à pas (DRAPER \& SMITH, 1966) permet de sélectionner parmi ces 9 termes ceux qui apportent le plus d'information pour expliquer le volume $v$, la variable dépendante.

Les résultats ainsi obtcnus donnent les tarifs $T_{2, A}, T_{2 . B}$ et $T_{2, C}$ applicables respectivement aux ensembles forestiers $\mathrm{A}, \mathrm{B}$ et $\mathrm{C}$ (cf. tabl. 2).

\subsubsection{Tests des tarifs}

On ne peut utiliser les valeurs des coefficients de détermination et des coefficients de variation pour comparcr les tarifs généraux aux tarifs spécifiques. En effet, ce type de critère de précision est bien adapté à la comparaison de tarifs différents, construits sur le même échantillon représentatif (PALM \& RondeuX, 1976). Mais ici, les tarifs généraux et les tarifs spécifiques sont calculés à partir d'échantillons différents qui n'ont donc pas les mêmes propriétés statistiques. It est préférable, dans ce cas, d'utiliser un critère basé sur l'analyse des erreurs de cubage résultant de l'emploi des tarifs sur des lots constitués d'arbres différents de ceux utilisés pour les construire (CAZE, 1978). A cet effet, nous avons tiré aléatoirement quatre lots différents de 100 arbres de chacun des ensembles forestiers A, B ct $C$. Nous avons appliqué à chaque lot les tarifs généraux et les tarifs spécifiques à une et à deux entrées. Nous avons déterminé, pour chaque lot, la moyenne relative des erreurs de cubage:

$$
\overline{\mathrm{e}}=\sum_{\mathrm{i}=1}^{100} \mathrm{e}_{\mathrm{i}} / 100 \cdot \mathrm{V}
$$

avec : $e_{i}=v_{i}-\hat{v}_{i}, i=1,2, \ldots ., 100$ où $v_{i}$ et $\hat{v}_{i}$ sont respectivement le volume exact et le volume estimé du ième arbre du lot et où $\bar{V}$ désigne le volume moyen exact des arbres du lot. cubage :

Nous avons également calculé l'écart type relatif de la distribution des erreurs de

$$
\sigma_{e}=\sum_{i=1}^{100}\left(e_{i}-\bar{e}\right)^{2} / 99 \cdot \bar{V}
$$

Pour chaque ensemble et pour chaque tarif, nous avons finalement reporté dans le tableau 3 les moyennes de ces valeurs relatives déterminées sur les quatre lots correspondants.

Pour les tarifs à une entréc, comme pour ceux à deux cntrées, on constate que l'utilisation des tarifs généraux pour les ensembles $\mathrm{A}$ et $\mathrm{B}$ donne un biais et une dispersion des erreurs de cubage toujours plus importants que lorsqu'on cube les lots provenant de ces ensembles avec les tarifs spécifiques. Remarquons également que les cubages des lots provenant des ensembles $\mathrm{A}$ et $\mathrm{B}$ par les tarifs spécifiques sont meilleurs que les cubages des lots provenant de l'ensemble $C$ par les tarifs généraux. 


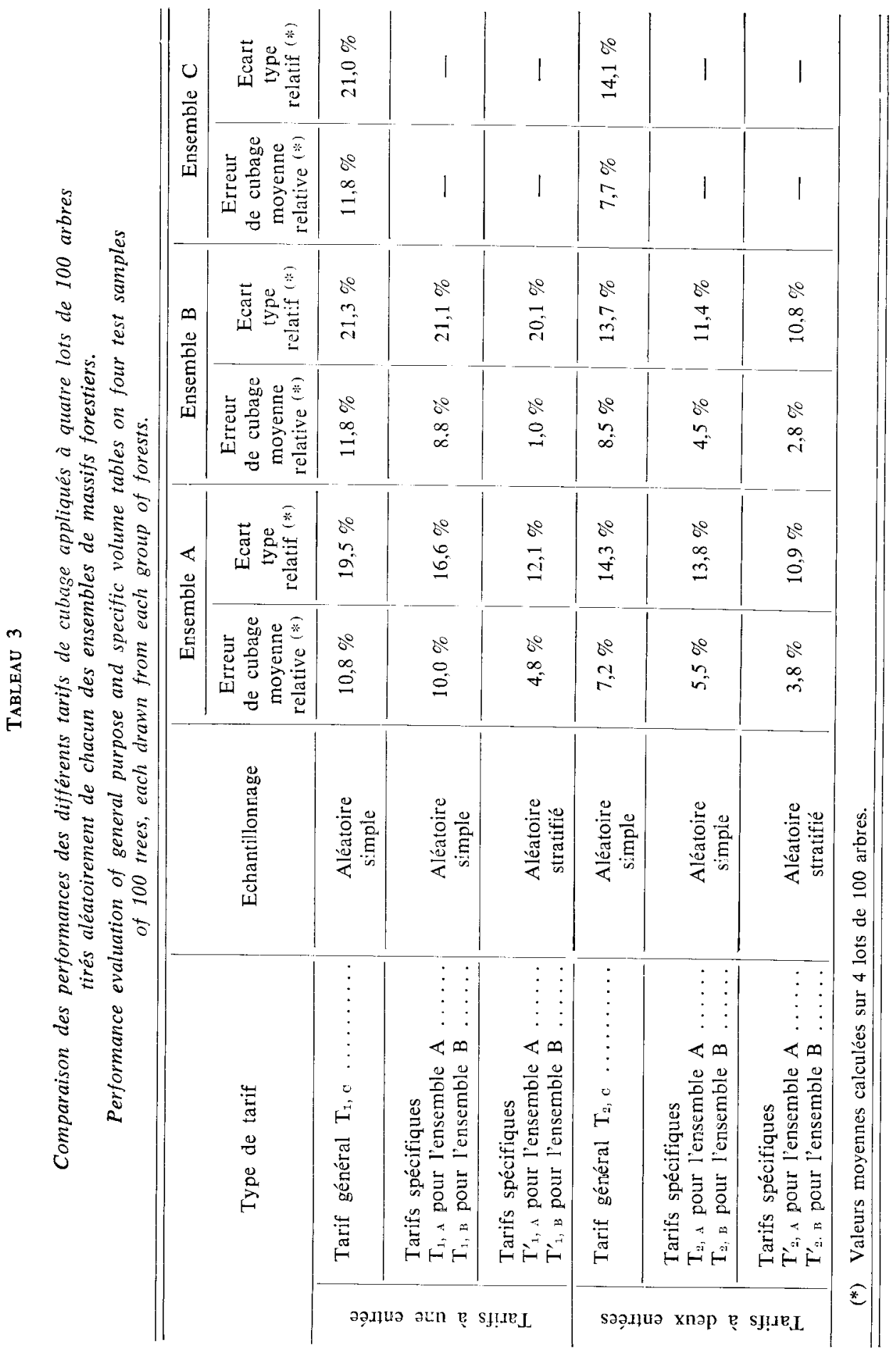


Les résultats que nous avons obtenus montrent l'intérêt pratique de construire des tarifs de cubage spécifiques aux ensembles de massifs forestiers au sein desquels les différents types de profil de cèdres présentent une relative stabilité.

Nous allons maintenant montrer qu'il est encore possible d'améliorer la précision des tarifs spécifiques en tenant compte de la répartition des cèdres entre les différents types de profil mis en évidence dans chacun des ensembles $\mathrm{A}$ et $\mathrm{B}$.

\subsection{Tarifs établis par échantillonnage stratifié}

Les tarifs spécifiques à une et à deux entrées ont été obtenus sans prendre en compte le polymorphisme naturel des cèdres. Or on sait que lorsque les individus dune population plus ou moins hétérogène peuvent être regroupés en sous-ensembles, ou strates, homogènes, on peut améliorer la représentativité de l'échantillon en utilisant une technique d'échantillonnage aléatoire stratifié (SNEdECOR \& Cochran, 1971).

L'échantillonnage stratifié est couramment utilisé en foresterie. Les distinctions entre les différentes strates s'y effectuent usuellement en fonction du milieu, du type de forêt (futaie, taillis, ...) ou encore de la densité du couvert (PARDE, 1961; Loetsch et al., 1973).

\subsubsection{Calcul des tarifs}

Nous nous proposons maintenant de stratifier l'échantillonnage des cèdres sur la base des trois profils-types mis en évidence précédemment. Les tarifs seront établis à partir d'échantillons constitués d'arbres représentatifs de trois profils-types, proportionnellement aux fréquences observées.

Pour chaque massif forestier, l'examen des distributions des diamètres à hauteur d'homme des arbres de chaque type de profil ne fait pas apparaître de corrélation entre la forme et la grosseur des tiges. L'échantillonnage est donc conduit de manière à ce que les arbres-échantillons de chaque type de profil aient des diamètres à hauteur d'homme distribués de la même manière que ceux de tous les arbres de l'ensemble forestier correspondant.

Les résultats de l'ajustement des équations de régression à une et à deux variables sur les échantillons stratifiés provenant des ensembles A et B sont donnés dans le tableau 2.

\subsubsection{Tests des tarifs}

Les tarifs spécifiques obtenus par échantillonnage stratifié ont été appliqués aux lots déjà utilisés pour tester les tarifs précédemment calculés. Les résultats de ces tests permettent d'affirmer que ces nouveaux tarifs sont nettement meilleurs que les tarifs construits par échantillonnage aléatoire simple, avec pourtant le même nombre d'arbreséchantillons (cf. tabl. 3). Cette supériorité se manifeste par une importante diminution du biais de l'estimation du volume des lots et par une réduction substantielle de la dispersion des erreurs de cubage. 


\section{Discussion et conclusions}

Le manque de précision de tarifs de cubage provient essentiellement des difficultés, pour une seulc équation de volume, d’être représentative de toutes les formes de tige qui peuvent être rencontrées dans un pcuplement forestier. Il est cependant possible d'accroître la précision des estimations de volume en affinant les techniques d'échantillonnage. En effet, nous avons mis au point une nouvelle méthodologie qui, basée sur des techniques de reconnaissance des formes, permet daméliorer la représentativité des échantillons servant au calcul des équations de volume. Grâce à de nombreux tests, il a été possible de chiffrer objectivement laccroissement sensible de précision et de fiabilité entraînés par l'application de cette méthodologie à l'inventaire et l'aménagement des forêts de cèdre du Rif au Maroc.

Nous n'avons pas voulu faire appel à des outils mathématiques sophistiqués pour modéliser la forme des arbres. Bien au contraire, nous avons recherché une caractérisation des profils qui soit aussi simple que possible et qui ne fasse appel qu'à des grandeurs couramment utilisées en foresterie. En réduisant chaque tige à un simple assemblage de trois troncs de cône, nous avons ainsi associé une observation quadridimensionnelle au profil de chaque arbre.

Nous avons alors concentré nos efforts sur lanalyse de la distribution de ces observations au sein des peuplements. De nouvelles techniques de classification automatique, basées sur une approche originale du problème de l'analyse des mélanges, ont permis de mettre en évidence trois types de profils de tige au sein de toutes les grandes forêts de cèdre du Maroc.

Pour tester la signification réelle de cette discrimination entre différents types de profils, nous avons entrepris la construction de différents tarifs de cubage pour les cédraies du Rif en établissant les plans d'échantillonnage en fonction des résultats de l'analyse des profils. Nous avons chiffré, par des tests, le gain de précision lié à l'emploi de chaque technique d'échantillonnage.

L'amélioration des tarifs de cubage a été obtenue en deux étapes successives. Dans un premier temps, nous avons regroupé les forêts en deux ensembles distincts au sein desquels les différents types de profil mis en évidence présentent une relative homogénéité. Les tarifs de cubage spécifiques, établis pour chacun des ensembles de forêts, donnent des résultats bien supérieurs à ceux obtenus avec des tarifs uniques, valables pour toutes les cédraies du Rif.

Dans un second temps, la représentativité des échantillons utilisćs pour construire les tarifs spécifiques a été améliorée par une technique de stratification respectant la distribution des tiges entre les trois profils-types mis en évidence. Les gains de précision par rapport aux résultats obtenus par échantillonnage aléatoire simple sont déterminants.

On pourrait objecter que si, à la place du diamètre à hauteur d'homme, on avait utilisé un diamètre mesuré à une hauteur relative de la tige $h / 10$, par exemple), ce paramètre fondamental aurait été mieux lié à la forme elle-même de l'arbre. Une telle modification dans la technique de caractérisation du profil des tiges améliorerait sans doute encore les résultats obtenus. Cependant, les difficultés pratiques rencontrées 
dans la mesure des diamètres à hauteur relative sont telles que nous avons été contraints à utiliser une mesure à hauteur fixe.

Finalement, il est très important de noter que l'utilisation de nos tarifs de cubage ne nécessite aucune mesure de forme sur le terrain. En ce sens, notre approche se différencie radicalement des méthodes d'inventaire qui demandent, en plus des mesures de diamètre à hauteur dhomme et de hauteur totale, la mesure d'un paramètre caractéristique de la forme des tiges (Behre, 1927 ; Spurr, 1952; Avery, 1967).

Nous n'avons présenté, dans cet article, que des résultats limités à une aire géographique restreinte et à une seule espèce forestière. Il serait souhaitable d'utiliser la méthodologie proposée pour construire des tarifs de cubage destinés à d’autres essences. Les résultats obtenus permettent également d'envisager l'application des principes exposés dans cette étude à l'élaboration d'autres fonctions tarifs. C'est dans cet esprit que nous tenterons d'améliorsr l'adéquation des fonctions de défilement qui permettent d'estimer les volumes à différentes hauteurs de découpe et présentent un intérêt évident pour la définition des assortiments.

Il résulte de cctte étude que de nouveaux progrès dans la construction des tarifs de cubage et, par suite, dans l'estimation des ressources forestières, peuvent être obtenus par une analyse approfondie de la forme des arbres. Il ne s'agit nullement de réduire, comme c'est souvent le cas, la forme à un seul paramètre. Le forestier se doit de considérer l'architecture de la tige qui ne peut être décrite et étudiée que par des techniques de reconnaissance des formes. Si la reconnaissance de la forme des tiges ne peut être envisagée dans la pratique forestière courante, il n’en reste pas moins vrai qu'au niveau de la construction des tarifs, les méthodes d'analyse multidimensionnelle peuvent contribuer efficacement à l'amélioration du plan d'échantillonnage et, par conséquent, à l'adéquation des fonctions tarifs.

\section{Remerciements}

Les auteurs voudraient remercier vivement pour leur appui et leur soutien dans la réalisation de ce travail :

- MM. ZAKi, Directeur des Eaux et Forêts et de la Conservation des Sols du Maroc;

- Vidal, Professeur à l'Université de Lille 1, France ;

- Vasseur, Chercheur au Centre d'Automatique de I'Université de Lille 1, France;

- NAJIM, Professeur à la Faculté des Sciences, Rabat, Maroc.

\section{Sumımary \\ Form analysis of trees for the construction of volume tables applied to Morocco-cedar (Cedrus atlantica Manetti)}

The volume equations which are used to construct volume tables are statistical relationships between the size of the trees and their stem form. Volume table construction for the Cedrus atlantica Manetti in Morocco has demonstrated the efficiency of pattern recognition techniques in forest inventory.

First, a multidimensional analysis of the form of more than 2000 felled trees has shown a marked polymorphism which has suggested to divide the forests of the Moroccan Rif mountains into two distinct groups. In each of them, three stable stem patterns have 
been identified. The objective differences that exist between the stem patterns of one group of forests and those of the other one have motivated the construction of specific volume tables for each group.

Field tests have demonstrated the improvement in accuracy gained by using two specific volume tables instead of a single general purpose one.

Finally, the volume estimation accuracy has been improved by means of a stratification by form class, thus taking into account the polymorphism of the stems within each group of forests. This stratified sampling strategy has appeared to be highly efficient in volume estimation.

Reçu le 14 décembre 1981.

Accepté le 25 mai 1982.

\section{Références bibliographiques}

Abadie J., Ayral P., 1956. Méthode de calcul du volume des peuplements sur pied dans les places d'essais de sylviculture. Ann. E.N.E.F., 1-135.

Avery T.E., 1967. Forest measurements. New York, St. Louis, 290 p.

Batchelor B.G., Wilkins B.R., 1969. Method for location of clusters of patterns to initialize a learning machine. Electron. Lett., 5 (20), 481-483.

Behre C.E., 1927. Form-class taper curves and volume tables and their application. J. Agric. Res., 35, 673-744.

BENZECRi J.P., 1978. L'allométric : étude des variations de forme liées à la taille chez un individu, une espèce ou plusieurs espèces. Les cahiers de l'analyse des données, $3(3), 371-376$.

Bouchon J., 1974. Les tarifs de cubage. Publication E.N.G.R.E.F., Nancy, 57 p.

BRENAC L., 1958. Corstruction d'un tarif de cubage. Rev. for, fr., $569-572$.

Bruce D., Curtis R.O., Vancoevfring D., 1968. Development of a system of taper and volume tables for red alder. For. Sci., 14 (3), 339-350.

Caze P., 1978. Méthodes de régression. I. - La régression sous contraintes. Les cahiers de l'analyse des données, 3 (2), 147-165.

Cochran W.G., 1963. Sampling techniques. Wiley, 2nd edition, Now York, 413 p.

Decouri N., 1965. Remarques sur la forme et la croissance des tiges. Rev. For. Fr., 512-524.

Dittmar O., 1958. Eine Studic tiber die «graphischen» Massencrmittlungsverfahren Speidelsche Massenkurve und Kopezky-Gehrhardtsche Massenlinic an Hand einer Kiefernkahisclag-Vollsektionierurg. Archiv Forstwes, 7 (2), 130-145.

Draper N., Simtн H., 1966. Applied regression analysis. John Wiley and Sons, New York, $407 \mathrm{p}$.

Duda R.O., HART P.E., 1973. Pattern classification and scene analysis. John Wiley and Sons, New York, Chapter 2, 482 p.

Fries J., MATERN B., 1965. On the use of multivariate methods for the construction of trec taper curves. I.U.F.R.O., Advis. Gr. For. Statit., Sect. 25, Conf. Stockholm R. Coll. For., Res. Notes 9, 85-117.

Hohenade W., 1924. Der Aufbau der Baumschäfte. Forstwiss. Centralbl., 46, 460-470.

Hummel F.C., 1955. The volume-basal area line. Bull. for. comn., London, 24, 84 p.

Jonson T., 1912. Taxatoriska undersökningar öfver skogsträdens form. III. - Formbestämning a staende träd. Skogsvardsför. Tidskr., 10 (4), 235-275.

Kozak A., Munro D.D., Smith J.H.G., 1969. Taper functions and their application in forest inventory. For. Chron., 45 (4), 278-283. 
LaRson P.R., 1963. Stem form development of forest trees. For. Sci., Monogr., 5 (Publ. Soc. Am. For.), 42 p.

Loetsch F., Zohrer F., Haller K.E., 1973. Forest. inventory. Vol. 2, BLV Verlagsgesellschaft, Munich, 469 p.

Mendiboure P., 1971. Equation de forme : méthode - premiers résultats. Ass. Forêt-Cellulose, Rapport annuel, 38-60.

Mendiboure P., Pronier G., 1971. Calcul des tarifs de cubage par régrcssion pondérée. Ass. Forêt-Cellulose, Rapport annuel, 11-29.

Naslund M., 1980. Stem form studies of pine in northern Sweden. Swed. Univ. Agric. Sc., Rapport $h^{\prime \prime} 8,86 \mathrm{p}$.

Palm R., 1981. Contribution méthodologique au cubage des arbres et à la construction de tables de cubage et d'assortiments. Thèse de Doctorat, Fac. Sci. agron., Gembloux, Belgique, $295 \mathrm{p}$.

Palm R., Rondeux J., 1976. A propos de deux problèmes rencontrés dans la construction de tarifs de cubage : le choix des équations de régression et l'échantillonnage des arbres. Bull. Rech. Agron. Gembloux, Belgique, 11, 243-258.

Papoulis A., 1965. Probability, random variables and stochastic processes. McGraw-Hill, New York, $583 \mathrm{p}$.

PaRdÉ J., Dendrométrie. E.N.E.F., Nancy, 350 p.

Postaire J.-G., Vasseur C., 1981. An approximate solution to normal mixture identification with application to unsupervised pattern classification. IEEE Trans. pattern analysis and machine intelligence, Vol. PAMI-3, 2, 163-179.

Postaire J.-G., 1981. Optimisation du processus de classification automatique par analyse de la convexité des fonctions de densité de probabilité. Application à la reconnaissance de la forme des arbres en dendrométrie. Thèse d'Etat, Université de Lille 1, $220 \mathrm{p}$.

Postaire J.-G., 1982. An unsupervised Bayes classifier for normal patterns based on marginal densities anlysis. Pattern Recognition, 15 (2), 103-111.

Postaire J.-G., VAsseur C., 1982. A fast algorithm for non-parametric probability density estimation. IEEE Trans. pattern analysis and machine intelligence. Vol. PAMI-4, $\mathrm{n}^{\circ} 6,663-666$.

Rondeux J., 1973. Principes de construction des tarifs de cubage mathématiques et de traitement automatique d'observations dendrométriques. Bull. Soc. R. For. Belgique, 80 (4), 165-187.

SChiffer A., 1905. Form und Inhalt des Lärche. Mitt. Forstl, Versuchsw. Osterr, Helft 31, $122 \mathrm{p}$.

Snedecor G.W., Cochran W.G., 1957. Statistical methods. Iowa State Univ. Press, Ames, U.S.A. (Traduction française de l'A.C.T.A., Paris, 1971, Méthodes statistiques, Chapitre $6,650 \mathrm{p}$.).

SpurR S.H., 1952. Forest inventory. Ronald Press Company, New York, 476 p. 